\title{
PREVALÊNCIA DE DISFUNÇÕES SEXUAIS ENTRE MULHERES ATENDIDAS EM UNIDADES DE SAÚDE DE CURITIBA
}

\author{
Caroline Schlossmacher,' Fernanda Rafaela Cabral Bonato, ${ }^{2}$ Lucas Schlossmacher ${ }^{3}$
}

PREVALENCE OF FEMALE SEXUAL DYSFUNCTIONS IN HEALTH UNITS IN CURITIBA

\section{PREVALENCIA DE DISFUNCIONES SEXUALES FEMENINAS EN UNIDADES DE SALUD DE CURITIBA}

Resumo: Esta pesquisa teve como objetivo verificar a prevalência de disfunções sexuais femininas em unidades de saúde de Curitiba. Como método foram utilizados uma anamnese clínica e sociodemográfica e o questionário Índice de Função Sexual Feminina (IFSF). Foram entrevistadas cem mulheres de quatro unidades de saúde. Para análise dos dados foi utilizado o programa Excel com análises estatísticas e de comparação. Como resultado encontrou-se prevalência de $36,8 \%$ de disfunções sexuais e de $49 \%$ de dificuldades sexuais, sendo que a média do escore do IFSF foi de 26,56 , valor próximo à nota de corte $(26,55)$. Os dados mostram que a dificuldade sexual mais prevalente foi relacionada ao desejo sexual. Verificou-se que existe relação de disfunções sexuais com idade e menopausa. O presente estudo constatou, ainda, o desconhecimento acerca da sexualidade e sobre disfunções sexuais na amostra estudada, principalmente frente a questão de tabus que permeiam a sexualidade. A partir do estudo, conclui-se significativa prevalência de disfunções e dificuldades sexuais em mulheres atendidas em unidades básicas de saúde de Curitiba, demonstrando quão importante é o trabalho de educação em sexualidade e intervenções clínicas para redução das queixas sexuais - especialmente na saúde pública.

Palavras-chave: sexualidade; saúde da mulher; saúde sexual; disfunção sexual; disfunção sexual feminina.

\begin{abstract}
This research aimed to verify the prevalence of female sexual dysfunction in health units in Curitiba. As method, a clinical and sociodemographic anamnesis was used and the Female Sexual Function Index (FSFI) questionnaire. One hundred women from four health units were interviewed. For data analysis, the Excel program was used with statistical and comparison analyzes. As a result, a prevalence of $36.8 \%$ of sexual dysfunctions and $49 \%$ of sexual difficulties was found, with the average of the FSFI score being 26.56, a value close to the cut-off score (26.55). The data show that the most prevalent sexual difficulty was related to sexual desire. It was found that there is a relationship of sexual dysfunction with age and menopause. The present study also found a lack of knowledge about sexuality and sexual dysfunctions in the sample studied, especially in view of the question of taboos about sexuality. From the study, a significant prevalence of sexual dysfunctions and difficulties was concluded in women attended in basic health units in Curitiba, demonstrating how important sexuality education work and clinical interventions are for reducing sexual complaints - especially in public health.
\end{abstract}

Keywords: sexuality; women's health; sexual health; sexual dysfunction; female sexual dysfunction.

Resumen: Esa investigación tuvo como objetivo verificar la prevalência de disfunciones sexuales femininas en las unidades de salude de Curitiba. Cómo método fue utilizado anamnese clínica y sociodemográfica y utilizado tambien el cuestionario Índice de la Funciión Sexual Feminina (ISFF). Fueron entrevistadas cien mujeres de cuatro unidades de salud distintas. Pra la análisis de datos fue utilizado el programa Excel, en las verificaciones de estatísticas y de comparación. Como resultado fue encontrado la prevalência de $36,8 \%$ de disfunciones sexuais y de $49 \%$ de dificuldades sexuales, teniendo por medio el la puntuación de IFSF fue de 26,56 , el valor aproximado de la nota de corte $(26,55)$. Los datos muestran que la dificultad sexual más prevalente fue relacionada al deseo sexual. Resultando aun existen relación de las disfunciones sexuales com la edad y la menopausia. El presente estudio constato todavía el desconocimiento acerca de

\footnotetext{
' Pós-graduação em Sexualidade Humana: educação e terapia pela Universidade Positivo (UP). Residência Multiprofissional em Saúde da Família da Secretaria Municipal de Saúde de Curitiba. E-mail: caroline.dsilveira@gmail.com

2 Doutoranda em Psicologia pela Universidade Federal do Paraná (UFPR). E-mail: fernandacbonato@gmail.com

${ }^{3}$ Acadêmico do $6^{\circ}$ ano de Medicina pela Universidade Positivo (UP). E-mail: schloss.lucas@gmail.com
} 
la sexualidade y las disfunciones sexuales en la amuestra estudiada, en principio frente a las cuestiones del tabu acerca de la sexualidade. En partida de eso concluye la gran prevalência de las disfunciones y dificultades sexuales de las mujeres servidas por el sistema básico de salud de Curitiba, quedando aclarado, así lo cuán importante es el trabajo em la educación em y para sexualidade y las intervenciones clínicas para la reducción de la quejas sexuales - en especial em la salud pública.

Palavras Claves: sexualidade; salud de la mujer; salud sexual; disfunción sexual; disfunción sexual feminina.

\section{Introdução}

A sexualidade é descrita pela Organização Mundial da Saúde (OMS) como um aspecto central do ser humano, o qual abrange sexo, identidades e papéis de gênero, orientação sexual, erotismo, prazer, intimidade e reprodução. Ela é vivenciada e expressa em pensamentos, fantasias, desejos, crenças, atitudes, valores, comportamentos, práticas, papéis e relacionamentos. Da mesma forma, a sexualidade é influenciada pela interação de fatores biológicos, psicológicos, sociais, econômicos, políticos, culturais, legais, históricos, religiosos e espirituais (ORGANIZAÇÃO MUNDIAL DA SAÚDE, 2006).

De acordo com a quinta edição do Manual Diagnóstico e Estatístico de Transtornos Mentais (DSM 5), disfunção sexual é definida como "um grupo heterogêneo de transtornos que, em geral, se caracterizam por uma perturbação clinicamente significativa na capacidade da pessoa em responder sexualmente ou de experimentar prazer sexual" (AMERICAN PSYCHOLOGICAL ASSOCIATION, 20I4, p. 423).

De modo geral, disfunções sexuais são caracterizadas pela falta, excesso, desconforto ou dor na expressão da resposta sexual, com sintomas há pelo menos seis meses e que cause sofrimento ao(a) paciente (ABDO, 2004b).

O referido manual demonstra, ainda, que a resposta sexual tem como essência a parte biológica, apesar de ser vivenciada em um contexto intra e interpessoal, que é permeado pela cultura. Assim, a função sexual envolve a complexa interação de fatores biológicos, socioculturais e psicológicos e, por isso, dificilmente é possível conhecer com exatidão a etiologia de um problema sexual. Além disso, o diagnóstico de uma disfunção sexual exige, necessariamente, a exclusão de problemas explicados por outras causas, como transtorno mental, efeitos de uma substância, condição de saúde, perturbação grave no relacionamento, violência do(a) parceiro(a) ou outros estressores (AMERICAN PSYCHOLOGICAL ASSOCIATION, 20I4).

Ademais, em relação a classificação das disfunções sexuais, elas podem ser classificadas em relação à: I) temporalidade (ao longo da vida ou adquirido); 2) circunstância (generalizada ou situacional); e 3) gravidade (leve, moderada ou grave) (AMERICAN PSYCHOLOGICAL ASSOCIATION, 20I4).
Além das disfunções sexuais, a definição de dificuldade sexual também se faz relevante no presente trabalho. Hayes e outros (2006) definiram o termo dificuldade sexual como um conceito mais geral relacionado à redução do funcionamento sexual, no qual a clareza do sofrimento sexual pode estar presente ou não.

Em relação à epidemiologia, o Estudo da Vida Sexual do Brasileiro constatou que a frequência de disfunções sexuais em mulheres foi de $50,9 \%$ e nos homens de $48,1 \%$; em relação às dificuldades sexuais, demonstrou que $28,5 \%$ das mulheres e $18,2 \%$ dos homens apresentaram alguma dificuldade sexual. Em contrapartida, o mesmo estudo verificou que, desta população com dificuldade sexual, $5,4 \%$ das mulheres e $7 \%$ dos homens realizaram tratamento (ABDO, 2004a). Outros estudos demonstraram que aproximadamente $45 \%$ das mulheres relataram ao menos uma queixa de disfunção sexual ao longo da vida (LEWIS et al., 2004; MCCABE et al., 20I6).

No que diz respeito à literatura científica nacional, não foram encontrados estudos similares a este, que se propusessem a avaliar a prevalência de disfunções sexuais femininas na atenção primária de Curitiba. Dessa forma, o presente estudo se faz relevante científica e socialmente. O objetivo da pesquisa foi verificar a prevalência de disfunções sexuais em mulheres cisgêneras, heterossexuais, homossexuais e bissexuais atendidas em unidades de saúde de Curitiba, na tentativa de sanar parte desta lacuna científica. Buscou-se também caracterizar e analisar questões da saúde sexual dessas mulheres.

\section{Método}

A pesquisa foi realizada em quatro unidades de saúde do Distrito Sanitário Portão (DSPO) de Curitiba. As participantes voluntárias, que aceitaram participar da pesquisa, totalizaram cem mulheres maiores de 18 anos atendidas nessas unidades de saúde.

Para fins de cálculo da amostra, foi considerado o número de mulheres com 18 anos ou mais com cadastro definitivo nas unidades de saúde em novembro de 2019 (34198) de acordo com os dados fornecidos pelo DSPO. Para o cálculo, foi utilizado o método de determinação da amostra para população finitas, com nível de confiabilidade de $95 \%$ e margem de erro de $10 \%$ (LEVINE; 
BERENSON; STEPHAN, 2000). A partir deste cálculo, o $\mathrm{N}$ da amostra foi definido como 96 , porém optou-se por considerar o número arredondado de cem participantes.

A coleta de dados foi realizada entre os meses de maio e outubro de 2020. As mulheres que estavam presentes nas unidades de saúde no momento da coleta foram convidadas a participar da pesquisa de forma livre e voluntária. $O$ convite foi realizado através de abordagem oral, informando que se tratava de uma pesquisa com mulheres e aquelas que aceitaram foram conduzidas a um dos consultórios da unidade de saúde, momento em que elas foram então informadas a respeito do tema da pesquisa. Dentre as mulheres abordadas, tentou-se evitar ao máximo um viés, de modo que quaisquer mulheres presentes na unidade de saúde no momento foram convidadas a participar.

Neste consultório em separado, a participante foi então devidamente informada sobre os objetivos e método da pesquisa e, após a assinatura do Termo de Consentimento Livre e Esclarecido (TCLE), a coleta de dados foi realizada. Para isso, as participantes responderam uma anamnese clínica e sociodemográfica e em seguida, foi aplicado o Índice de Função Sexual Feminina (IFSF), tendo duração de aproximadamente 20 minutos por participante. Ambos, a anamnese e o questionário, foram aplicados de forma oral, para contemplar pessoas que eventualmente não soubessem ler ou escrever.

Todas as cem mulheres participantes do estudo responderam a anamnese clínica e sociodemográfica para fins de caracterização da amostra e definição da data da última relação sexual. Em relação à aplicação do questionário foram excluídas mulheres sem atividade sexual nas últimas quatro semanas, uma vez que o instrumento utilizado avalia a função sexual apenas nesse período. Dessa forma, foi utilizado o critério de exclusão apenas para a resposta do questionário e não para a participação do estudo.

A anamnese foi realizada de forma semiestruturada, de modo que havia perguntas fechadas, mas também havia espaço para que as mulheres falassem de modo aberto sobre suas respostas. Através da anamnese foram contempladas questões sociodemográficas e clínicas, tais como: idade, etnia, religião, escolaridade, ocupação, orientação sexual, estado civil, idade da primeira relação sexual, histórico de relacionamento, se a mulher tinha filhos ou não, tipo de parto, uso de substâncias psicoativas, comorbidades, medicação em uso, histórico de cirurgias, método contraceptivo, se a mulher estava ou não na menopausa, motivo pelo qual veio até a unidade de saúde, sintomas/ queixas relacionadas à saúde mental, se a mulher já teve alguma dificuldade e/ou disfunção sexual, se já realizou algum tipo de tratamento para dificuldade e/ou disfunção sexual, se algum profissional da saúde já havia perguntado sobre disfunção e/ou dificuldade sexual e se a mulher já falou para alguém sobre sua dificuldade/disfunção sexual.
O IFSF se caracteriza por ser um questionário breve e multidimensional, de referência internacional, traduzido e validado para o Brasil (THIEL, 2008). Seu principal objetivo é avaliar a resposta sexual feminina nas últimas quatro semanas, considerando seus domínios: desejo sexual, lubrificação vaginal, orgasmo, satisfação sexual e dor. Ao final, obtém-se um escore relacionado a cada um dos domínios, que é multiplicado por um fator homogeneizador, sendo que o escore final pode variar de 2 a 36 (PACAGNELLA; MARTINEZ; VIEIRA, 2009; THIEL 2008). O instrumento original não apresenta um escore de corte delimitado, porém, estudos definiram tal escore como 26,55 , sendo que valores iguais ou abaixo desse escore indicariam disfunção sexual (SUTTER LATORRE et al., 20I5).

Para análise dos resultados foi utilizado o programa Excel com as análises comparativas de: distribuição, média, desvio-padrão, mediana, intervalo interquartil, teste $\mathrm{R}$ de Pearson e qui-quadrado.

É importante frisar que a coleta de dados foi realizada em 2020, durante a pandemia de COVID-19 que acometeu $\circ$ mundo. Realizar essa pesquisa em meio a pandemia foi um verdadeiro desafio, principalmente em função da significativa redução de fluxo de pacientes nas unidades de saúde. Todavia, todas as medidas e cuidados necessários foram realizados para preservar a saúde e bem-estar das participantes e pesquisadores. Desta forma, é possível considerar que o estresse gerado pela pandemia, isolamento social e outras dificuldades decorrentes podem ter apresentado influência sobre a qualidade de vida e sexualidade da população, incluindo as voluntárias. 


\section{Resultados e discussão}

$\mathrm{Na}$ Tabela I a seguir estão demonstrados os dados sociodemográficos das participantes da pesquisa.

Tabela I - Dados sociodemográficos

\section{Tipo de informação}

Idade

\begin{tabular}{|c|c|c|}
\hline Média & 39,6 & \\
\hline Amplitude (min-máx) & $18-77$ & \\
\hline Desvio padrão & 15,2 & \\
\hline Etnia $\mathbf{N}$ & & $\%$ \\
\hline Branca & 69 & $69 \%$ \\
\hline Parda & 24 & $24 \%$ \\
\hline Preta & 6 & $6 \%$ \\
\hline Asiática & I & $1 \%$ \\
\hline
\end{tabular}

\begin{tabular}{l|l|l}
\hline Heterossexual & 94 & $94 \%$ \\
\hline Homossexual & 2 & $2 \%$ \\
\hline Bissexual & 4 & $4 \%$
\end{tabular}

Eestado civil

\begin{tabular}{|c|c|c|}
\hline Solteira & 50 & $50 \%$ \\
\hline Casada & 34 & $34 \%$ \\
\hline Divorciada & 10 & $10 \%$ \\
\hline União estável & 4 & $4 \%$ \\
\hline Viúva & 2 & $2 \%$ \\
\hline \multicolumn{3}{|l|}{ Religião } \\
\hline Católica & 44 & $44 \%$ \\
\hline Evangélica & 30 & $30 \%$ \\
\hline Espírita & 8 & $8 \%$ \\
\hline Umbandista & 2 & $2 \%$ \\
\hline Testemunha de Jeová & 2 & $2 \%$ \\
\hline Mórmon & 1 & $1 \%$ \\
\hline Sem religião & 13 & $13 \%$ \\
\hline \multicolumn{3}{|l|}{ Escolaridade } \\
\hline Ensino superior completo & 20 & $20 \%$ \\
\hline Ensino superior incompleto & 12 & $12 \%$ \\
\hline Ensino médio completo & 32 & $32 \%$ \\
\hline Ensino médio incompleto & 9 & $9 \%$ \\
\hline Ensino fundamental completo & 4 & $4 \%$ \\
\hline Ensino fundamental incompleto & 23 & $23 \%$ \\
\hline
\end{tabular}

Fonte: elaborada pelos autores (2020). 
Dentre as mulheres entrevistadas, 68 (68\%) haviam tido relação sexual nos últimos 30 dias e 32 (32\%) não, sendo que as últimas responderam apenas a anamnese e não o questionário IFSF por critério de exclusão, conforme exposto anteriormente. $O$ menor tempo da última relação sexual foi zero dia e o maior 25 anos.

Dentre toda a amostra, foi constatado que 49 (49\%) mulheres referiram ter alguma dificuldade sexual, enquanto $3(3 \%)$ delas já haviam recebido diagnóstico de disfunção sexual por profissional de saúde, entretanto, 16 (16\%) já haviam realizado algum tratamento para esta condição.

A partir do IFSF, a média do escore total das entrevistadas foi 26,56 , valor próximo à nota de corte $(26,55)$, dentre as mulheres que responderam ao questionário, $37 \%$ apresentaram disfunção sexual e $63 \%$ não.

$\mathrm{Na}$ Figura I, a seguir, foi realizada a comparação dos principais resultados obtidos na pesquisa.

Figura I - Comparação dos resultados

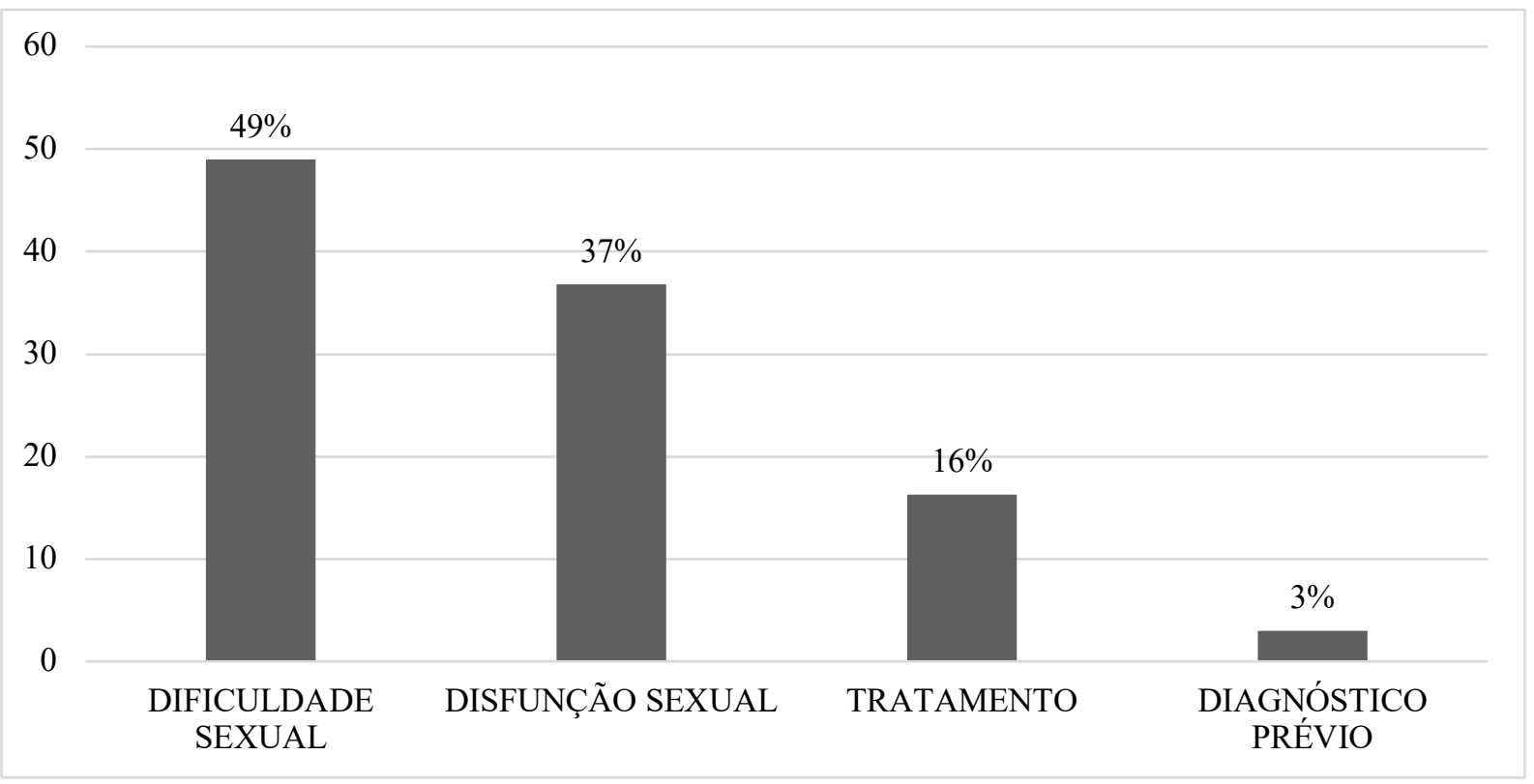

Fonte: elaborada pelos autores (202I).

As médias dos escores parciais do IFSF são apresentadas na Tabela 2. Entre as queixas de dificuldades sexuais apresentadas, a mais frequente foi baixa libido, o que corrobora com os achados demonstrados pelo escore do instrumento.

Tabela 2 - Média dos escores parciais e geral

\begin{tabular}{l|l|l|l|l}
\hline Domínio & Escore & Desvio padrão (DP) & Mínimo (MIN) & Máximo (MAX) \\
\hline Desejo & 3,44 & 1,06 & 1,20 & 6,00 \\
\hline Excitação & 4,10 & 1,06 & 1,20 & 6,00 \\
\hline Lubrificação & 4,69 & 1,09 & 1,50 & 6,00 \\
\hline Orgasmo & 4,54 & 1,22 & 1,20 & 6,00 \\
\hline Satisfação & 4,91 & 1,13 & 1,20 & 6,00 \\
\hline Dor & 4,89 & 1,44 & 0 & 6,00 \\
\hline Total & 26,56 & 5,3 & 12 & 35,3 \\
\hline
\end{tabular}

Fonte: elaborada pelos autores (2020).

A média da idade das mulheres que apresentaram disfunção sexual foi de 4I,I anos (DP= I5,5; Min=18; $M a x=68)$, enquanto a idade média daquelas que não apresentaram disfunção sexual foi de 33 anos (DP = I I 6;
Min= 18; Max=55). Esses dados sugerem possível correlação entre idade e disfunção sexual, hipótese reforçada por meio do teste $\mathrm{R}$ de Pearson, o qual retornou valor $-0,40$, evidenciando uma associação linear inversa 
intermediária. Ou seja, foi verificada que há uma tendência de que mulheres com idade avançada apresentem nota mais baixa no escore do IFSF.

Ao todo foram entrevistadas 30 mulheres na menopausa, sendo que metade delas estavam no critério de exclusão; dentre as avaliadas, $67 \%$ apresentaram disfunção sexual e $33 \%$ não. Com os dados obtidos, verificou-se relação entre menopausa e disfunção sexual, confirmada pelo teste qui-quadrado ( $\mathrm{p}$ valor $<0,0 \mathrm{I}$ ).

Em relação ao uso de antidepressivos e associação com disfunção sexual, pelo teste qui-quadrado, foi encontrado $p$ valor de $0, I$ I. Com isso, não foi possível afirmar se existe relação significativa entre disfunção sexual e uso de antidepressivos. Em contrapartida, $40 \%$ das mulheres com disfunção sexual relataram alguma queixa de saúde mental.

Enfim, considerando a amostra total, $71 \%$ das mulheres nunca haviam sido perguntadas sobre dificuldade sexual por algum(a) profissional de saúde. Enquanto dentre aquelas com disfunção sexual, $24 \%$ foram perguntadas sobre dificuldade sexual por algum(a) profissional de saúde e $40 \%$ relataram sua dificuldade para alguém (parceria, médico(a), familiares, amigo(a), psicólogo(a)).

A prevalência de disfunções sexuais femininas verificada na pesquisa foi de $37 \%$, enquanto $3 \%$ das mulheres relataram diagnóstico prévio. Esses dados assemelham-se ao estudo de Ferreira, Souza e Amorim (2007) realizado no Recife, o qual relatou prevalência de $37 \%$ de disfunções sexuais em mulheres. Dados do National Health and Social Life Survey (NHSLS) corroboram com os encontrados na presente pesquisa, segundo os quais entre 30 e $50 \%$ das mulheres americanas apresentavam disfunção sexual (SALONIA et al., 2004). Tal dado aproximou-se também da prevalência apresentada por McCabe e outros (2016) que demonstraram que a prevalência de mulheres que reportaram ao menos uma disfunção sexual foi de aproximadamente 40 a 50\%. De modo similar, Lewis e outros (2004) constataram que 40 a $45 \%$ das mulheres adultas apresentaram ao menos uma manifestação de disfunção sexual. Em contrapartida, o Estudo da Vida Sexual do Brasileiro demonstrou que $50,9 \%$ das mulheres apresentam disfunção sexual (ABDO, 2004a).

A presente pesquisa demonstrou que $49 \%$ das entrevistadas relataram alguma dificuldade sexual. Enquanto alguns estudos aproximaram-se deste dado, outros achados distanciaram-se do resultado obtido. Shifren e outros (2008) constataram prevalência de $44,2 \%$ de dificuldade sexual entre mulheres norte-americanas. Em contrapartida, Smith e outros (2012) demonstraram que $36 \%$ das mulheres australianas relataram ao menos uma queixa de dificuldade sexual no último ano, enquanto Abdo (2004a) verificou que $28,5 \%$ das mulheres brasileiras apresentam alguma dificuldade sexual.

Percebe-se que, dentre as entrevistadas, $16 \%$ realizaram algum tipo de tratamento para dificuldade e/ou disfunção sexual, entretanto, o estudo de Abdo (2004a) verificou que $5,4 \%$ das mulheres realizaram tratamento para alguma dificuldade sexual.

Este estudo possibilitou verificar variabilidade entre os números apresentados e os estudos supracitados. Isso se deve a fatores, como: variação na definição de disfunção sexual, amostra da população, método para determinar presença de disfunção, idade, questões culturais e método da coleta de dados (MCCABE et al., 2016). Apesar da discrepância, não se pode negar que os estudos supracitados demonstraram prevalência significativa de dificuldades e disfunções sexuais femininas.

A partir dos dados obtidos, percebe-se que o número de mulheres que já foram perguntadas sobre sexualidade por profissionais de saúde não é alto (29\%). Além disso, o número de diagnósticos das disfunções sexuais é baixo (3\%). A esse respeito, pode-se pensar que isso se deve ao desconhecimento relacionado à sexualidade, ao fato de que muitos profissionais não têm, em suas formações, matérias e abordagens específicas de sexualidade, além da desatualização sobre o tema (BONATO, 2019; MARQUES; CHEDID; EIZERIK, 20I2).

Segundo Abdo e Fleury (2006), muitas vezes as disfunções sexuais femininas não são devidamente diagnosticadas por inibição da paciente (que não relata ou nem reconhece a queixa) ou do(a/e) profissional de saúde (que, por vezes, não investiga a função sexual da paciente). Entretanto, o diagnóstico de disfunções sexuais é de extrema relevância devido a interferência na qualidade de vida e associação a outras questões de saúde que podem estar relacionadas. Além disso, a avaliação da função sexual da mulher pode, também, auxiliar na identificação de possíveis conflitos psicológicos, de relacionamento e outras questões de saúde mental, assim, a investigação clínica de rotina mostra-se necessária (ABDO; FLEURY, 2006).

Em relação à idade e prevalência de disfunção sexual, o presente estudo identificou que a idade média das mulheres que apresentaram disfunção sexual foi de $4 I, I$ anos, enquanto aquelas que não apresentaram disfunção sexual tiveram idade média de 33 anos. Assim, observa-se que a presença de disfunção sexual é proporcional à idade, conforme constatado por McCabe e outros (2016) e Ricci e Ambrósio (2019).

As modificações orgânicas que ocorrem na mulher durante o climatério e a menopausa podem influenciar a resposta sexual, tornando-a mais lenta nesse período. Alterações como ressecamento e hipotrofia vaginal, dor à penetração e sensação de ardor são frequentes na menopausa. Em relação a comportamentos sexuais como o desejo sexual e o orgasmo, tem-se uma influência multifatorial que envolve aspectos psicológicos, socioculturais, interpessoais e biológicos (BRASIL, 2008).

Dentre as mulheres que estavam na menopausa, metade não havia tido relação sexual no último mês. 
A esse respeito, sabe-se que os sintomas da menopausa - principalmente de ordem sexual - podem acarretar em diminuição da frequência sexual. Além disso, os presentes dados validaram a relação entre menopausa e disfunção sexual, conforme indicado pela literatura científica (PENTEADO et al., 2000; RICCl; AMBRÓSIO, 20I9).

McCabe e outros (2016) demonstraram, ainda, que problemas de desejo e excitação foram os mais frequentes entre as mulheres com disfunção sexual, o que corrobora com dados verificados, pois a média do escore parcial de desejo foi a menor dentre todas, além da queixa de baixa de libido ter sido a mais frequente dentre as dificuldades sexuais relatadas.

Ademais, $40 \%$ das mulheres que apresentaram disfunção sexual relataram alguma queixa de saúde mental. Tal dado corrobora com a literatura, trazendo à tona os significativos prejuízos na qualidade de vida que a disfunção sexual pode causar (ORGANIZAÇÃO MUNDIAL DA SAÚDE, 2002; WORLD HEALTH ORGANIZATION QUALITY OF LIFE GROUP, 1994), como sofrimento emocional/ mental, (ABDO, 2007; CAVALCANTI; CAVALCANTI, 2006), ansiedade (CAVALCANTI; CAVALCANTI, 2006), sentimento de culpa, baixa autoestima, sintomas depressivos e depressão (ABDO, 2007).

Por fim, é importante salientar que para a seleção das participantes não houve distinção em relação a identidade de gênero (cisgêneras/transgêneras) nem de orientação sexual (heterossexual/homossexual/ bissexual/assexual e pansexual), porém, $100 \%$ das mulheres voluntárias da pesquisa se autodeclararam mulheres cisgêneras e a maioria delas (94\%) heterossexuais.

A esse respeito, foi constatado durante as entrevistas que $o$ instrumento utilizado contempla mulheres heterossexuais em relacionamentos estáveis. Isso ficou claro a partir da utilização de termos como: parceiro apenas no pronome masculino, penetração do pênis na vagina (sem considerar sextoys ou penetração anal), além de limitar a relação sexual à entrada de pênis na vagina (não considerando outras formas de relação sexual como sexo oral ou práticas não penetrativas), sendo que a sexualidade está além disso (ORGANIZAÇÃO MUNDIAL DA SAÚDE, 2006). Sobre isso, Puppo (2012) ressalta que apesar do IFSF ser a escala mais utilizada para avaliar a disfunção sexual em mulheres, ele parece estar essencialmente focado na função orgástica resultante da relação pênis-vagina. Dessa forma, deixa de considerar questões como o orgasmo feminino ser causado pelos órgãos eréteis femininos, ou seja, clitóris, bulbos e pequenos lábios. Além disso, fazer sexo pode se referir a atos que envolvem duas pessoas, como sexo vaginal, sexo anal, sexo oral ou masturbação mútua. $O$ autor defende ainda o conceito de "fazer sexo/fazer amor" como a situação em que o orgasmo acontece em ambos os parceiros(a) com ou sem relação sexual: uma definição para todos os seres humanos (PUPPO, 20I2).

Faz-se importante citar estes fatos, porque eles são relevantes para a análise dos dados expostos, uma vez que seguem uma lógica heteronormativa e cisgênera, presente na sociedade, que exclui e/ou traz invisibilidade às pessoas com orientação ou identidade de gênero distinta da cisheterossexualidade.

\section{Conclusão}

A partir da realização deste estudo, concluiu-se que há prevalência significativa de dificuldades e disfunções sexuais na população em questão. Também foi possível constatar, através dos relatos das participantes, que há desconhecimento a respeito de disfunções sexuais, uma vez que mulheres desconheciam esse conceito, além daquelas que convivem com este sofrimento sem saber que algo está errado. Dessa forma, evidenciou-se a necessidade de abordagens multiprofissionais na identificação e tratamento de disfunções sexuais.

O diagnóstico de disfunção sexual apresentou relevância ao considerar suas perturbações na qualidade de vida e na saúde das mulheres. Assim, colocar a sexualidade em pauta pode ser benéfico no sentido de auxiliar na identificação de possíveis problemas biológicos, psicológicos e/ou sociais. Fica evidenciada então a relevância dessa abordagem durante as consultas.

A pesquisa demonstrou baixa frequência de perguntas de profissionais de saúde sobre sexualidade, dificuldades ou disfunções sexuais. A partir disso, percebe-se que, na maioria das vezes, a saúde sexual da mulher não está sendo abordada nas unidades de saúde. A esse respeito, levantou-se a hipótese de desconhecimento, por parte dos(as/es) profissionais, sobre sexualidade e disfunções sexuais. Com isso, percebe-se a necessidade de capacitações referentes ao tema para que possam acolher e tratar essas mulheres de forma acolhedora, adequada, ética e com embasamento técnico/científico.

Por fim, ressalta-se a necessidade de ações e políticas públicas voltadas para reduzir este quantitativo de queixas sexuais a partir de estratégias como educação em sexualidade, intervenções clínicas e capacitação dos profissionais. Para tal, faz-se necessário mais e maiores pesquisas a respeito, de modo que essa população possa ter maior acesso à informação de qualidade, assistência à saúde e tratamentos necessários. 


\section{Referências}

ABDO, C. H. N. Estudo da vida sexual do brasileiro (ESVB). São Paulo: Bregantini, 2004a.

ABDO, C. H. N. Descobrimento sexual do Brasil. São Paulo: Summus, 2004b.

ABDO, C. H. N. Da depressão à disfunção sexual (e vice-versa). In: ABDO, C. H. N. Da depressão à disfunção sexual (e vice-versa). São Paulo: Segmento Farma, 2007.

ABDO, C. H. N.; FLEURY, H. J. Aspectos diagnósticos e terapêuticos das disfunções sexuais femininas. Archives of Clinical Psychiatry, São Paulo, v. 33, n. 3, p. 162-167, 2006.

AMERICAN PSYCHIATRIC ASSOCIATION. DSM-5: Manual diagnóstico e estatístico de transtornos mentais. Porto Alegre: Artmed Editora, 2014.

BONATO, F. R. C. A formação científica sobre sexualidade nos cursos de graduação em psicologia da região de Curitiba. 2019. Dissertação (Mestrado em Psicologia) - Setor de Ciências Humanas, Universidade Federal do Paraná, Curitiba, 2019.

BRASIL. Ministério da Saúde. Manual de atenção à mulher no climatério/menopausa. Brasília, DF: Editor do Ministério da Saúde, 2008.

CAVALCANTI, R.; CAVALCANTI, M. Tratamento clínico das inadequações sexuais. 3. ed. São Paulo: Editora Roca, 2006.

FERREIRA, A. L. C. G.; SOUZA, A. I; AMORIM, M. M. R. Prevalência das disfunções sexuais femininas em clínica de planejamento familiar de um hospital escola no Recife, Pernambuco. Revista Brasileira de Saúde Materno Infantil, Recife, v. 7, n. 2, p. I43-I50, 2007.

HAYES, R. D. et al. Epidemiology: What can prevalence studies tell us about female sexual difficulty and dysfunction?. The Journal of Sexual Medicine, Amsterdam, v. 3, n. 4, p. 589-595, 2006.

LEVINE, D. M.; BERENSON, M. L.; STEPHAN, D. Estatística: teoria e aplicações. Rio de Janeiro: LTC, 2000.
LEWIS, R. W. et al. Epidemiology/risk factors of sexual dysfunction. The Journal of Sexual Medicine, Amsterdam, v. I, n. I, p. 35-39, 2004.

MARQUES, F. Z. C.; CHEDID, S. B.; EIZERIK, G. C. Resposta sexual humana. Revista de Ciências Médicas, Campinas, v. 17, n. 3/6, 2012.

MCCABE, M. P. et al. Incidence and prevalence of sexual dysfunction in women and men: a consensus statement from the Fourth International Consultation on Sexual Medicine 2015. The Journal of Sexual Medicine, Amsterdam, v. I3, n. 2, p. I44-I52, 2016.

ORGANIZAÇÃO MUNDIAL DA SAÚDE. Gender and reproductive rights 4 . Geneva, Switzerland: World Health Organization, 2002. Disponível em: www.who.int/reproductive-health/gender/ glossary.html

\section{ORGANIZAÇÃO MUNDIAL DA SAÚDE.}

Defining sexual health: Report of a technical consultation on sexual health. Geneva, Switzerland: World Health Organization, 2006.

PACAGNELLA, R. C.; MARTINEZ, E. Z.; VIEIRA, E. M. Validade de construto de uma versão em português do Female Sexual Function Index. Cadernos de Saúde Pública, Rio de Janeiro, v. 25, 2333-2344, 2009.

PENTEADO, S. R. L. et al. Sexualidade no climatério e na senilidade. Revista de Ginecologia e Obstetrícia, [s. I.], v. II, n. 3, p. I88-192, 2000.

PUPPO, V. Female sexual function index (FSFI) does not assess female sexual function. Acta Obstetricia et Gynecologica Scandinavica, [s. I.], v. 9I, n. 6, p. 759-759, 2012.

RICCI, M. D.; AMBRÓSIO, A. Sexualidade e câncer de mama. Barueri: Manole, 2019.

SALONIA, A. et al. Women's sexual dysfunction: a pathophysiological review. BJU International, Oxford, v. 93, n. 8, p. II56-II 64, 2004.

SHIFREN, J. L. et al. Sexual problems and distress in United States women: prevalence and correlates. Obstetrics \& gynecology, Hagerstown, MD, v. II2, n. 5, p. 970-978, 2008. 
38 Revista Brasileira de

Sexualidade Humana

DOI: https://doi.org/10.35919/rbsh.v32il.961

SMITH, A. M. A. et al. Incidence and persistence/ recurrence of women's sexual difficulties:

Findings from the Australian longitudinal study of health and relationships. Journal of Sex \& Marital Therapy, London, v. 38, n. 4, p. 378-393, 2012.

SUTTER LATORRE, G. F. et al. Escores de corte para o FSFI. Revista Inspirar Movimento \& Saude, [s. I.], v. 7, n. I, 2015.

THIEL, R. R. C. Tradução, adaptação cultural e validação do Female Sexual Function Index (FSFI) para língua portuguesa. 2008. Tese (Doutorado em Cirurgia) - Faculdade de Ciências Médicas, Universidade Estadual de Campinas, Campinas, 2008. Disponível em: http://www.repositorio. unicamp.br/handle/REPOSIP/3 I I 596.

WORLD HEALTH ORGANIZATION QUALITY OF LIFE GROUP (WHOQOL GROUP).

The development of the World Health Organization quality of life assessment instrument (the WHOQOL). In: ORLEY, J; KUYKEN, W. (ed.). Quality of life assessment: international perspectives. Heidelberg: Springer Verlag, 1994.

p. $4 \mathrm{I}-60$. 